\title{
Lazaroa
}

ISSN-e 1988-3307

\section{Index of nomenclatural syntaxonomic novelties}

Catapodio marini-Hymenolobetum revelierii Bioret, Delbosc \& Panaïotis ass. nova ....

Catapodio marini-Centaurietum tenuiflori Bioret, Delbosc \& Panaïotis ass. nova ........

Helichryso italici-Thymeleaetum tartonrairae Bioret, Delbosc, Panaïotis \& Paradis

ass. nova

Helichryso italici-Thymeleaetum tartonrairae schoenetosum nigricans Bioret,

Delbosc, Panaïotis \& Paradis subass. nova

Helichryso italici-Thymeleaetum tartonrairae dianthetosum siculi Bioret, Delbosc \&

Panaïotis subass. nova

Loto cytisoidis-Juncetum acuti Bioret, Delbosc \& Panaïotis ass. nova

Loto cytisoidis-Dactyletum hispanicae crithmetosum maritimae Bioret, Delbosc \&

Panaïotis subass. nova

Chaerophyllo hirsuti-Betuletum pubescentis Rodríguez-Guitián, Real, Romero Franco \& Álvarez-Hurtado ass. nova

Valeriano officinalis-Betuletum pubescentis Rodríguez-Guitián, Real, Romero Franco \& Álvarez-Hurtado ass. nova

Lonicero implexae-Quercion cocciferae Meddour, Meddour-Sahar, Zeraia \& Mucina all. nova

Genisto quadriflorae-Pinetum halepensis Meddour, Meddour-Sahar, Zeraia \& Mucina ass. nova

Smilaco asperae-Pistacietum lentisci (Nègre 1964) Meddour, Meddour-Sahar, Zeraia \& Mucina nom. nov.

Genisto tricuspidatae-Calicotomion spinosi Dahmani-Megrerouche \& Loisel ex Meddour,

Meddour-Sahar, Zeraia \& Mucina all. nova

Calicotomo spinosae-Quercetum rotundifoliae Dahmani-Megrerouche \& Loisel ex Meddour, Meddour-Sahar, Zeraia \& Mucina ass. nova

Cisto salviifolii-Quercetum rotundifoliae Dahmani-Megrerouche \& Loisel ex Meddour,

Meddour-Sahar, Zeraia \& Mucina ass. nova

Calicotomo intermediae-Quercion cocciferae Dahmani-Megrerouche \& Loisel ex

Meddour, Meddour-Sahar, Zeraia \& Mucina all. nova

Calicotomo intermediae-Quercetum rotundifoliae Dahmani-Megrerouche \& Loisel ex Meddour, Meddour-Sahar, Zeraia \& Mucina ass. nova

Loto dorycnium-Quercion rotundifoliae Djebaïli in Meddour, Meddour-Sahar, Zeraia

\& Mucina all. nova

Loto dorycnium-Pinetum halepensis Djebailli, Meddour \& Mucina in Meddour, Meddour-

Sahar, Zeraia \& Mucina ass. nova

Coronillo valentinae-Quercetum Guinochet ex Meddour in Meddour, Meddour-Sahar,

Zeraia \& Mucina ass. nova

Ephedro majoris-Juniperetalia phoeniceae Quézel \& Barbero ex Meddour, Meddour-

Sahar, Zeraia \& Mucina ord. novo 
Lamio garganici-Cedrion atlanticae Abdessemed in Meddour, Meddour-Sahar, Zeraia \& Mucina all. nova

Ranunculo aurasiaci-Cedretum atlanticae Meddour, Meddour-Sahar, Zeraia \& Mucina ass. nova

Abietion maroccano-numidicae Mucina \& Meddour in Meddour, Meddour-Sahar, Zeraia \& Mucina all. nova

Plagio maghrebini-Quercion canariensis Meddour, Meddour-Sahar, Zeraia \& Mucina all. nova

Plagio maghrebini-Quercetum canariensis Laribi in Meddour ex Meddour, MeddourSahar, Zeraia \& Mucina ass. nova

Viburno tini-Quercetum canariensis Meddour, Meddour-Sahar, Zeraia \& Mucina ass. nova

Quercetum balloto-broteroi Meddour, Meddour-Sahar, Zeraia \& Mucina ass. nova ......

Scrophulario laevigatae-Acerion obtusati Meddour, Meddour-Sahar, Zeraia \& Mucina all. nova

Scrophulario laevigatae-Aceretum obtusati Wojterski ex Meddour, Meddour-Sahar,

Zeraia \& Mucina ass. nova

Clematido cirrhosae-Populion albae Bensettiti \& Lacoste ex Meddour, Meddour-Sahar, Zeraia \& Mucina all. nova

Salici pedicellatae-Fraxinion angustifoliae Meddour, Meddour-Sahar, Zeraia \& Mucina all. nova

Equiseto maximi-Fraxinetum angustifoliae Bensettiti \& Lacoste ex Meddour, MeddourSahar, Zeraia \& Mucina ass. nova

Campanulo alatae-Alnion glutinosae Meddour, Meddour-Sahar, Zeraia \& Mucina all. nova

Viti viniferae-Alnetum glutinosae Meddour, Meddour-Sahar, Zeraia \& Mucina ass. nova ...

Tamo communis-Salicion atrocinereae de Foucault \& Julve ex Mucina \& Meddour in Meddour, Meddour-Sahar, Zeraia \& Mucina all. nova

Nerio oleandri-Tamaricetum Meddour-Sahar, Zeraia \& Mucina ass. nova

Donatio fascicularis-Schoenetum antarctici Amigo, San Martín, Ramírez \& Álvarez ass. nova

Nanodeo muscosae-Sphagnetum magellanici Amigo, San Martín, Ramírez \& Álvarez ass. nova

Abrotanello linearifoliae-Bolacion caespitosae Amigo, San Martín, Ramírez \& Álvarez all. nova

Senecio trifurcati-Bolacetum caespitosae Amigo, San Martín, Ramírez \& Álvarez ass. nova

Azorello selaginis-Bolacetum caespitosae Amigo, San Martín, Ramírez \& Álvarez ass. nova

Halimiono portulacoidis-Salicornietum perennis (M. Fernández \& A. Santos 1984)

Del Arco, Izco \& O. Rodríguez nom. nov.

Halimiono portulacoidis-Salicornietum perennis subass. typicum Del Arco, Izco \& O.

Rodríguez subass. nova.

Halimiono portulacoidis-Salicornietum perennis subass. limonietosum canariensis

(M. Fernández \& A. Santos 1984) Del Arco, Izco \& O. Rodríguez, comb. nov. 